\title{
Étude de l'effet de diverses espèces de plantes des prairies permanentes sur l'hydrolyse enzymatique des constituants pariétaux
}

\author{
J Scehovic \\ Station fédérale de recherches agronomiques de Changins, $\mathrm{CH} 1260$ Nyon, Suisse
}

(Reçu le 6 décembre 1993; accepté le 20 mai 1994)

\begin{abstract}
Résumé - Une méthode simple, rapide et répétable a été mise au point pour estimer le potentiel de diverses espèces de plantes des prairies d'inhiber la dégradation enzymatique de la fraction pariétale des cellules végétales, facteur déterminant la digestibilité de la matière organique. Les substances susceptibles d'inhiber l'hydrolyse enzymatique des polyosides pariétaux ont été extraites à chaud à l'aide d'un tampon phospho-citrique $(\mathrm{pH} 4,6)$ à partir d'un échantillon de Geranium silvaticum. L'indice d'action négative potentielle (IANP) indique l'importance de cette action exercée par l'extrait végétal sur la cellulolyse enzymatique, comparativement à celle enregistrée en l'absence de l'extrait. Le niveau de dégradation des glucides pariétaux a été quantifié par la quantité d'acides phénoliques libérés de leurs liaisons (esters) avec les constituants pariétaux sous l'action de la cellulase. La détermination de I'IANP pour de nombreuses plantes des prairies permanentes a révélé des différences importantes entre les principaux groupes d'espèces (Graminées, Légumineuses, autre plantes) et entre espèces à l'intérieur de ces groupes. Cet indice peut aider à déterminer la proportion acceptable d'une espèce dans une association végétale.
\end{abstract}

cellulolyse / cellulase / dégradation / inhibition / phénols

Summary - A study of the influence of different plant species from natural grasslands on enzymatic hydrolysis of cell-wall polysaccharides. A simple, rapid and repetitive method for the estimation of the potential of herbage to inhibit enzymatic activity is described. The enzymatic degradabit ity of the cell walls (the factor determining the organic matter digestibility) was quantified by the phenolic compounds liberated by cellulase action of their ester linkages with cell-wall carbohydrates. Inhibition of this action by some plant compounds solubilised during refluxing in a phosphocitric buffer solution $(\mathrm{pH}$ 4.6) was expressed as an index of potential negative action (IANP). This index expresses the action of inhibitory compounds in plant extract on the cellulolytic activity of a fungal enzyme as compared with the activity of this enzyme measured without plant inhibitors. This is useful in the prediction of the potential negative action of plant species on the quality of the herbage association of which they are a part. The determination of IANP in many natural grassland species shows important differences between grass, legumes and other dicotyledonous plants and also within these botanical groups. This index can be useful for the determination of an acceptable proportion of individual plant species in herbages.

cellulolyse / cellulase / degradability / inhibition / phenols 


\section{INTRODUCTION}

Les prairies naturelles ou permanentes assurent une part importante de l'alimentation des ruminants. La qualité du fourrage produit par ces associations végétales dépend essentiellement du mode d'exploitation (fréquence des coupes, alternance fauchepâture, niveau de fertilisation, etc). Les différentes techniques d'exploitation des prairies influencent fortement leur composition floristique. Les proportions respectives des Graminées, Légumineuses et autres Dicotylédones dans les fourrages déterminent leur composition chimique et, à travers celleci, leur valeur nutritive et alimentaire. La proportion parfois élevée de certaines Dicotylédones, riches en métabolites secondaires, peut être responsable de la qualité médiocre d'un fourrage (Scehovic, 1988, 1990, 1991). La digestibilité, déterminée par les techniques de laboratoire, de la majorité des Dicotylédones est relativement élevée par suite de leur faible teneur en constituants pariétaux. Leur forte concentration en métabolites secondaires, inhibiteurs potentiels de l'activité enzymatique et microbienne du rumen, peut être néfaste pour la digestibilité des autres espèces, riches en constituants pariétaux (Graminées), présentes dans la même association végétale.

L'action antinutritionnelle de certains métabolites secondaires et particulièrement celle exercée par des substances phénoliques peut se manifester de plusieurs manières :

- augmentation de la résistance des parois cellulaires à l'action des enzymes (Swain, 1977 ; Hartley et Jones, 1977; Beart et al, 1985) ;

- inhibition de l'activité cellulolytique des enzymes (Daiber, 1975 ; Griffiths et Jones, 1977) ;

- inhibition de la production des enzymes par les micro-organismes du rumen (action bactéricide et bactériostatique) (Cheng et al, 1980 ; Chesson et al, 1982 ; Borneman et al, 1986 ; Jung, 1988).
L'action la plus préjudiciable pour la qualité du fourrage est celle qui a pour résultat l'inhibition des enzymes cellulolytiques (Swain, 1977 ; Mueller-Harvey, 1989). Nous avons essayé, à l'aide d'une technique de laboratoire, de quantifier cette action et de l'exprimer sous forme d'un indice appelé «indice de l'action négative potentielle» (IANP).

Dans l'interprétation des valeurs obtenues, il faut tenir compte du fait que l'inhibition des enzymes observée in vitro tend à surestimer l'action des inhibiteurs observée in vivo. Les répercussions effectives d'une action inhibitive peuvent se manifester si les barrières naturelles ou les mécanismes de détoxitication existant dans le tube digestif des ruminants sont surchargés par la concentration élevée des métabolites secondaires dans le fourrage ingéré (Singleton et Kratzer, 1969 ; Hagerman et Butler, 1991).

\section{MATÉRIEL ET MÉTHODES}

\section{Principe de la méthode}

Au cours de l'hydrolyse des polyosides pariétaux, les enzymes cellulolytiques ont la capacité de libérer les acides phénoliques de leurs liaisons esters qui les attachent aux polyosides pariétaux (Hartley et Jones, 1977; Borneman et al, 1986). Ce processus peut être entravé par la présence dans le milieu de substances inhibitrices de l'activité cellulolytique des enzymes. II suffit alors, après l'hydrolyse, de mesurer la quantité de phénols solubles libérés dans l'hydrolysat en présence des inhibiteurs et de la comparer avec la concentration des phénols libérés en absence d'inhibiteurs (témoin).

\section{Matière végétale}

Pour étudier la faisabilité, mettre au point la procédure analytique et tester ses possibilités (rendement journalier et répétabilité), il a fallu d'une part extraire les substances inhibitrices potentielles à partir d'une espèce végétale connue pour 
son action antinutritionnelle, d'autre part isoler un substrat correspondant à la fraction fibreuse de la matière végétale exempte de tous phénols solubles et riche en acides phénoliques estérifiés, libérables par les enzymes cellulolytiques. Dans ce contexte, nous avons choisi Geranium silvaticum, espèce particulièrement riche en composés phénologiques, biologiquement très actifs (Scehovic, 1990). Le substrat fibreux a été isolé à partir de Festuca arundinacea, espèce très riche en acides phénoliques (Scehovic, 1979).

\section{Extraction des «inhibiteurs»}

Deux g de matière (Geranium silvaticum) séchée à $55^{\circ} \mathrm{C}$ dans une étuve ventilée et réduite en poudre $(1 \mathrm{~mm})$ ont été soumis à une ébullition sous reflux (1 h) dans une solution tampon phospho-citrique (Jones et Hayward, 1973). Le tampon est un mélange de 2 solutions aqueuses dont une contient $35,59 \mathrm{~g} / /$ d'hydrogénophosphate de di-sodium dihydrate (solution $A$ ) et la deuxième $21,01 \mathrm{~g} / \mathrm{l}$ d'acide citrique monohydrate (solution B). Le mélange des 2 solutions $(875 \mathrm{ml} \mathrm{A+}$ $1000 \mathrm{ml} \mathrm{B}$ ) donne la solution tampon dont le $\mathrm{pH}$ doit être de 4,6.

Après ébullition et refroidissement, l'extrait contenant des substances inhibitrices a été séparé du résidu par filtration (filtre plissé rapide). La teneur en phénols solubles de Geranium silvaticum, mesurée à l'aide du réactif de Folin-Ciocalteu (Scehovic, 1990) était de 7,29\% dans la matière sèche, dont la fraction polymérisée représente $57,1 \%$.

L'utilisation du réactif de Folin-Ciocalteu, malgré sa spécificité relative, est justifiée par la rapidité, la répétabilité et le taible coût du procédé (Mueller-Harvey, 1969). En accord avec la suggestion de Hagerman et Buttler (1991), les concentrations des phénols déterminées par cette technique colorimétrique doivent être considérées comme «apparentes».

\section{Isolement du substrat fibreux}

Trente $\mathrm{g}$ de matière (Festuca arundinacea) séchée à l'étuve $\left(24 \mathrm{~h}\right.$ à $\left.55^{\circ} \mathrm{C}\right)$ et réduite en poudre $(1 \mathrm{~mm})$ ont été soumis à une ébullition sous reflux $(1,30 \mathrm{~h})$ dans $1,5 \mathrm{I}$ d'un solvant composé d'un mélange méthanol/eau (2:1 vol/vol) acidifié (1\%) avec l'acide chlorhydrique. Le résidu a été séparé de l'extrait par filtration (verre fritté porosité $N^{\circ} 1$ ) et réextrait dans 1 I d'acétone pa (30 min sous reflux). Le résidu-substrat a été filtré et lavé à l'acétone jusqu'à l'élimination des pigments, puis séché $\left(55^{\circ} \mathrm{C}\right)$.

La teneur du substrat en acides phénoliques estérifiés déterminée selon Scehovic (1990) était de $2,54 \%$. Notons que n'importe quelle Graminée dépassant le stade de l'épiaison peut servir de matière de base pour la préparation du substrat.

\section{Inhibition de l'activité cellulolytique de l'enzyme cellulase par l'extrait de Geranium silvaticum}

Dans une série de tubes Sovirel (fermeture à vis), on a introduit $200 \mathrm{mg}$ de substrat et $10 \mathrm{ml}$ d'un mélange composé de la solution tampon phospho-citrique $(A+B)$ et des quantités croissantes de l'extrait de Geranium silvaticum. Les phénols apportés par l'extrait correspondent à des quantités de 0 à $14,7 \mathrm{mg} / \mathrm{ml}$ du milieu enzymatique.

Le mélange tampon/extrait a été additionné par le même volume de l'enzyme cellulase (Aspergillus niger-0,67 U/mg, Fluka) dissoute dans la solution tampon $(A+B)$ à raison de $12,5 \mathrm{U} / \mathrm{ml}$. Les concentrations des phénols libérés du substrat par l'action de la cellulase correspondent à la différence entre la concentration globale des substances phénoliques après l'incubation avec la cellulase et les phénols apportés par l'extrait et l'enzyme. Afin de pouvoir calculer ces concentrations, chaque procédé a été accompagné d'un témoin (extrait + enzyme) sans substrat.

Les échantillons et leur témoin respectif ont été incubés pendant $24 \mathrm{~h}$ (agitation permanente à $39^{\circ} \mathrm{C} \pm 0,1^{\circ} \mathrm{C}$ ). Après l'incubation, l'hydrolysat a été séparé du résidu du substrat par filtration à travers des creusets filtrants (verre fritté porosité $N^{\circ} 1$ ) préalablement séchés et tarés. La perte de masse du substrat, au cours de la cellulolyse, a été déterminée gravimétriquement. La concentration des phénols dans l'hydrolysat a été déterminée à l'aide du réactif de Folin-Ciocalteu (Scehovic, 1990).

\section{RÉSULTATS}

Le dosage des phénols solubles dans le mélange enzyme/extrait (sans substrat) 
après $24 \mathrm{~h}$ d'incubation montre leur diminution par rapport à leur concentration avant incubation (jusqu'à 16\%) (fig 1). Cette diminution correspond à la somme des phénols de l'extrait et de ceux, dosés comme tels, de l'enzyme rendus insolubles par la complexation de l'enzyme par des phénols de l'extrait. L'inhibition de l'activité de l'enzyme est due au blocage du site actif de la protéine-enzyme dont la responsabilité chez Geranium silvaticum incombe probablement aux polymères phénoliques. Chez les autres espèces végétales, pauvres en phénols polymérisés, l'action similaire (blocage) peut être développée par différents inhibiteurs potentiels (terpènes, alcaloïdes et autres).

Cette inhibition de l'activité cellulolytique de l'enzyme a pour résultat la diminution de la dégradabilité du substrat et par conséquent la diminution de la quantité des phénols solubilisés (acides phénoliques libérés du substrat) (fig 2). La corrélation entre ces 2 paramètres est très étroite $(R=0,99)$. La solubilité partielle du substrat dans la cellulase inactivée (fig 2) est due à sa solubilité dans le tampon (20\%) et au fait que l'inhibition totale de l'activité enzymatique n'est pas immédiate après le contact avec les inhibiteurs.

À partir du rapport entre la teneur en acides phénologiques libérés du substrat (dosés comme phénols solubles totaux) en l'absence de l'extrait (témoins) et ceux libérés en présence de l'extrait (inhibiteurs), on peut calculer l'inhibition du processus de l'hydrolyse enzymatique.

\section{Adaptation du procédé pour l'analyse de routine}

L'analyse de plusieurs dizaines d'espèces participant à la composition floristique des

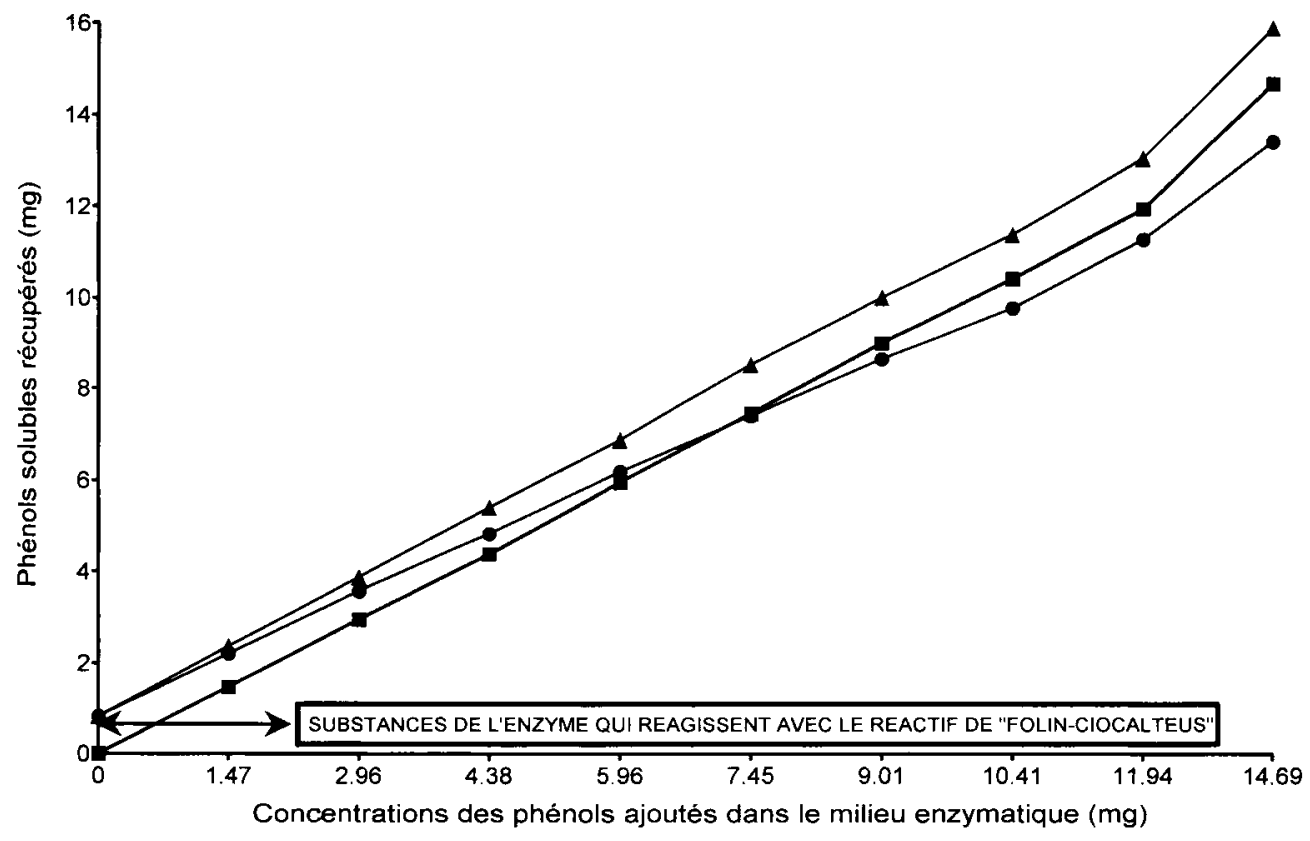

Fig 1. Concentration des phénols dans le milieu (sans substrat). - - phénols ajoutés (extrait); -A- avant incubation ; - - après incubation. 


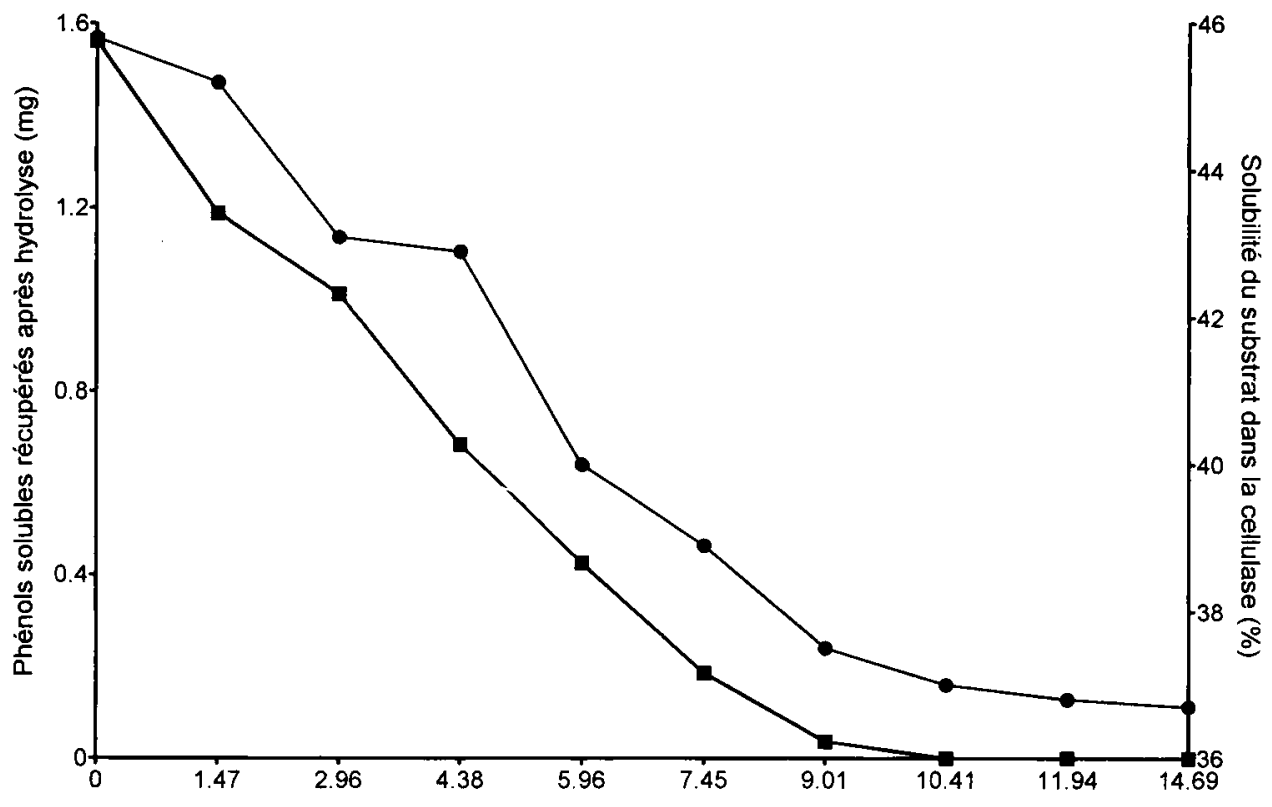

Concentrations des phénols ajoutés dans le milieu enzymatique (mg)

Fig 2. Évolution du niveau de dégradation du substrat en relation avec la concentration des phénols du milieu. - - phénols libérés du substrat ; - - solubilité (tampon + cellulase) du substrat.

prairies permanentes, effectuée dans notre laboratoire, démontre la présence plus ou moins importante des inhibiteurs potentiels dans la majorité des Dicotylédones. II est intéressant d'exprimer cette potentialité sous forme d'un paramètre de laboratoire.

Le paramètre ne fait appel qu'à une méthodologie simple, rapide et très répétable (tableau I), sans exigences particulières en matériel et produits chimiques.

On procède comme suit : $100 \mathrm{mg} \mathrm{du}$ substrat fibreux préparé comme décrit plus haut sont introduits dans des tubes Sovirel (bouchon à vis) de $10 \mathrm{ml}$. Ajouter $5 \mathrm{ml}$ de l'extrait de l'échantillon ( $1 \mathrm{~h}$ d'ébullition sous reflux de $1 \mathrm{~g}$ d'échantillon dans $50 \mathrm{ml}$ de la solution tampon) et $5 \mathrm{ml} \cdot$ de la solution de cellulase (préparation du tampon et cellulase comme décrit plus haut). Chaque échantillon (extrait) est accompagné du
Tableau I. Test de répétabilité (analyse effectuée 10 fois sur la même matière : Taraxacum officinale).

\begin{tabular}{cc}
$\begin{array}{c}\text { Phénols libérés } \\
\text { (spectrométrie) } \\
(m g)\end{array}$ & $\begin{array}{c}\text { Solubilité cellulase } \\
\text { (gravimétrie) } \\
(\%)\end{array}$ \\
\hline 0,943 & \\
0,946 & 44,6 \\
0,940 & 45,8 \\
0,943 & 45,4 \\
0,946 & 46,2 \\
0,944 & 45,3 \\
0,944 & 46,8 \\
0,947 & 44,7 \\
0,945 & 46,5 \\
0,943 & 44,5 \\
0,002 & 45,3 \\
0,21 & 0,803 \\
& 1,76
\end{tabular}


témoin (extrait + enzyme - sans substrat) qui sert à estimer la quantité de phénols apportés par leurs constituants. L'activité cellulolytique de l'enzyme (sans inhibition) est déterminée à l'aide de témoins (faits en double) où on remplace l'extrait par la solution tampon. Les échantillons et les témoins sont placés dans un incubateur à $39^{\circ} \mathrm{C}$ $\left( \pm 0,1^{\circ} \mathrm{C}\right)$ et agités continuellement. Après $24 \mathrm{~h}$ d'incubation, les tubes sont centrifugés (10 min à $7000 \mathrm{t} / \mathrm{min}$ ). Une partie aliquote du surnageant est introduite dans une fiole jaugée de $100 \mathrm{ml}$. Une heure après l'addition de carbonate de sodium (20\%) et du réactif de Folin-Ciocalteu, la mesure de la densité optique $(675 \mathrm{~nm})$ est effectuée à l'aide d'un spectrophotomètre (Scehovic, 1990).

\section{Calculs de l'indice de l'action négative potentielle (IANP)}

La valeur de l'indice exprime l'importance de l'action inhibitrice d'un échantillon sur la dégradation des matières fibreuses du substrat, mesurée à l'aide des acides phénologiques libérés lors de la cellulolyse enzymatique.

$$
\text { IANP }=100-(100 \mathrm{PLE} / \mathrm{PLT})
$$

où $\mathrm{PLE}=$ phénols libérés en présence de l'extrait [(extrait + enzyme + substrat) (extrait + enzyme) $;$ PLT = phénols libérés sans extrait (témoin) [(tampon + enzyme + substrat) - (tampon + enzyme)] ; nombre $100=$ paramètre de correction permettant que l'IANP $\geq 0$.

Les variations de l'IANP sont conditionnées par les relations suivantes:

$\begin{array}{lc}\text { Relation } & \text { IANP } \\ \text { PLE }=\text { PLT } & 0 \\ \text { PLE } \leq \text { PLT } & \leq 100 \\ \text { PLE } \leq 0 & \geq 100\end{array}$

\section{RÉSULTATS ET DISCUSSION}

L'estimation d'IANP de plusieurs dizaines d'espèces présentes dans les prairies naturelles montre que de nombreuses espèces peuvent développer une action fortement négative sur la cellulolyse des autres végétaux présents dans la même association (tableau II). Malgré leur propre digestibilité parfois très élevée (tableau II), la présence de ces espèces en quantité importante dans l'association végétale peut avoir des conséquences néfastes pour la qualité de l'échantillon global. Le fait que les espèces dont I'IANP est élevée (tableau II) soient mal consommées ou même refusées par les ruminants indique une certaine relation entre cet indice et l'acceptabilité ou l'appétibilité des plantes de prairies. II est intéressant de noter que certaines espèces se distinguent par un IANP toujours très élevé, indépendamment de leur âge, de la saison et du lieu de prélèvement et que d'autres présentent des différences intraspécifiques très importantes en relation avec les critères agronomiques (tableau III). Les valeurs d'IANP dépassant 100 (PLE $\leq 0$, voir calculs de l'IANP) laissent supposer, en accord avec les observations de Swain (1977, 1979), Hartley et Jones (1977), Van Soest (1981), Beart et al (1985), Barry (1989), Scehovic (1990), qu'une partie des phénols actifs forme des liaisons non seulement avec les protéines de l'enzyme, mais aussi avec les polymères du substrat (polyosides pariétaux) (fig 3). Cette action, conjuguée à celle effectuée sur les enzymes, pourrait augmenter encore le potentiel d'inhibition de la digestibilité développée par les végétaux concernés.

Une étude préliminaire effectuée dans notre laboratoire sur une vingtaine d'échantillons (prairies permanentes riches en espèces) testés préalablement avec des animaux a montré que I'IANP associé à quelques autres critères chimiques peut corriger les différences entre la digestibilité in 
Tableau II. IANP et digestibilité estimée (DMOL, Scehovic, 1991) de quelques espèces prélevées dans des prairies permanentes ( 1 échantillon par espèce).

\begin{tabular}{|c|c|c|}
\hline Espèces & IANP & $\begin{array}{c}D M O L \\
\%\end{array}$ \\
\hline \multicolumn{3}{|l|}{ Dicotylédones } \\
\hline Hypericum perforatum & 285 & 79,4 \\
\hline Geranium silvaticum & 234 & 80,2 \\
\hline Alchemilla vulgaris & 224 & 78,5 \\
\hline Salvia pratensis & 193 & 70,4 \\
\hline Leontodon hispidus & 184 & 73,7 \\
\hline Taraxacum officinale & 174 & 82,7 \\
\hline Centaurea jacea & 136 & 61,2 \\
\hline Rumex obtusifolius & 136 & 72,0 \\
\hline Plantago lanceolata & 125 & 80,1 \\
\hline Achillea millefolium & 123 & 61,5 \\
\hline Galium mollugo & 103 & 74,6 \\
\hline Centaurea montana & 103 & 82,2 \\
\hline Polygonum bistorta & 101 & 68,2 \\
\hline Lotus corniculatus & 100 & 69,4 \\
\hline Chrysanthemum leucanthemum & $m 96$ & 67,9 \\
\hline Heracleum sphondylium & 89 & 72,0 \\
\hline Rumex acetosa & 88 & 66,0 \\
\hline Crepis biennis & 81 & 63,2 \\
\hline Knautia arvensis & 77 & 59,4 \\
\hline Silene vulgaris & 76 & 77,6 \\
\hline Anthriscus silvestris & 68 & 65,0 \\
\hline Trifolium pratense & 66 & 70,7 \\
\hline Ranunculus friesianus & 64 & 61,1 \\
\hline Medicago sativa & 48 & 64,5 \\
\hline Onobrychis viciifolia & 43 & 62,2 \\
\hline Trifolium repens & 39 & 75,7 \\
\hline \multicolumn{3}{|l|}{ Graminées } \\
\hline Dactylis glomerata & 64 & 75,8 \\
\hline Agropyron repens & 54 & 69,5 \\
\hline Festuca rubra & 41 & 56,7 \\
\hline Holcus lanatus & 40 & 60,2 \\
\hline Brachypodium pinnatum & 38 & 54,7 \\
\hline Phleum pratense & 34 & 67,6 \\
\hline Poa trivialis & 33 & 65,5 \\
\hline Festuca pratensis & 33 & 62,1 \\
\hline Bromus mollis & 32 & 67,6 \\
\hline Bromus erectus & 22 & 64,5 \\
\hline Poa pratensis & 21 & 65,3 \\
\hline Lolium perenne & 17 & 67,1 \\
\hline
\end{tabular}

DMOL\% $=101,259-0,72 \mathrm{LC}-1,295 \mathrm{~L}+0,272 \mathrm{CV}-$ $6,390 \mathrm{CPFI}$ où LC : lignocetlulose ; L : lignine ; CV : cellulose vraie ; $\mathrm{CPFl}$ : phénols insolubles «attachés" aux polyosides pariétaux.
Tableau III. Exemples de variation intraspécifique de I'IANP.

\begin{tabular}{lrrr} 
Espèces & \multicolumn{3}{c}{ Échantillons } \\
\cline { 2 - 4 } & 1 & 2 & 3 \\
& & & \\
& & & \\
Alchemilla vulgaris & 194 & 231 & 190 \\
Geranium silvaticum & 234 & 220 & 208 \\
Leontodon hispidus & 153 & 158 & 155 \\
Plantago lanceolata & 125 & 125 & 125 \\
Salvia pratensis & 192 & 193 & 243 \\
Sanguisorba minor & 200 & 219 & 220 \\
& & & \\
Anthriscus silvestris & 54 & 105 & 150 \\
Lotus corniculatus & 39 & 77 & 100 \\
Onobrychis vicifolia & 43 & 57 & 78 \\
Taraxacum officinale & 51 & 101 & 174 \\
Trifolium pratense & 54 & 75 & 120 \\
Trifolium repens & 34 & 49 & 63 \\
& & &
\end{tabular}

vivo et celle estimée par les techniques de laboratoire (fig 4). Cela doit être confirmé encore sur un nombre plus important d'échantillons (testés in vivo) et surtout sur ceux riches en Dicotylédones non Légumineuses.

Le but principal de cette étude a été de proposer un test de caractérisation des espèces végétales relativement peu connues du point de vue alimentaire, constituant la population floristique des herbages naturels. La composition chimique usuelle (azote, fractions des parois ou du contenu cellulaire, etc) n'est qu'une description très superficielle de la qualité de la matière végétale analysée. Un critère de laboratoire, indiquant le potentiel de plantes des prairies (particulièrement des Dicotylédones) d'influencer le processus de dégradation enzymatique des constituants pariétaux, peut contribuer à une meilleure définition de la qualité des fourrages estimée à l'aide de techniques de laboratoire.

L'indice d'action négative potentielle s'accorde avec cet objectif. II est toutefois 


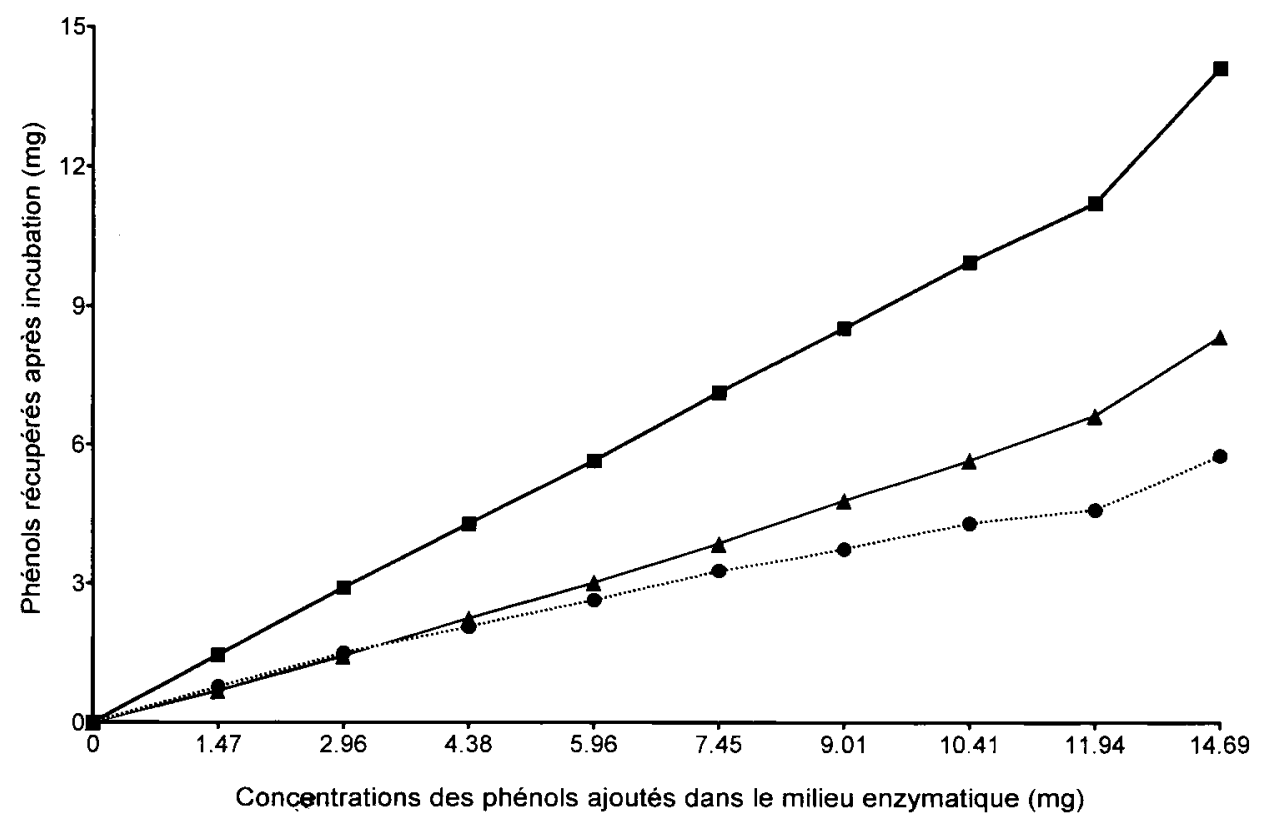

Fig 3. Interaction entre les phénols de l'extrait (Geranium silvaticum) et le substrat fibreux (sans

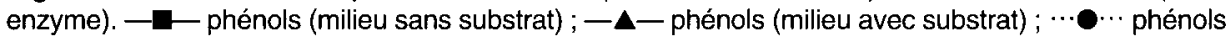
"attachés" aux polyosides pariétaux du substrat.

nécessaire de rappeler qu'il ne s'agit que d'un indice dont l'interprétation doit être accompagnée d'un certain nombre de réserves tenant compte des différences importantes entre l'environnement très complexe du tube digestif et celui relativement simple et standardisé conçu pour l'expérimentation in vitro.

L'intérêt principal de la méthode proposée consiste dans l'utilisation de I'IANP comme critère d'appréciation de la qualité du fourrage. L'estimation de la dégradation des composés pariétaux à l'aide des phénols libérés par l'enzyme par rapport à la méthode gravimétrique généralement utilisée pour l'estimation de la digestibilité des fourrages (Jones et Hayward, 1973 ; Hartley et al, 1974 ; Aufrère, 1982) est selon notre expérience plus rapide (30 échan- tillons/j), plus précise et plus répétable (tableau 1).

\section{CONCLUSIONS}

Plusieurs années d'étude de l'estimation de la qualité des fourrages par des méthodes de laboratoire nous amènent à la conclusion que la concentration de substances individuelles dans la matière végétale n'est qu'une valeur indicative de la qualité. Cela est valable surtout dans le domaine des métabolites secondaires. Effectivement, il est impossible d'expliquer les effets de l'une ou l'autre des substances inhibitrices potentielles de la digestion sans connaître leur «activité anti-biologique» qui n'est pas forcément proportionnelle à leur concentra- 


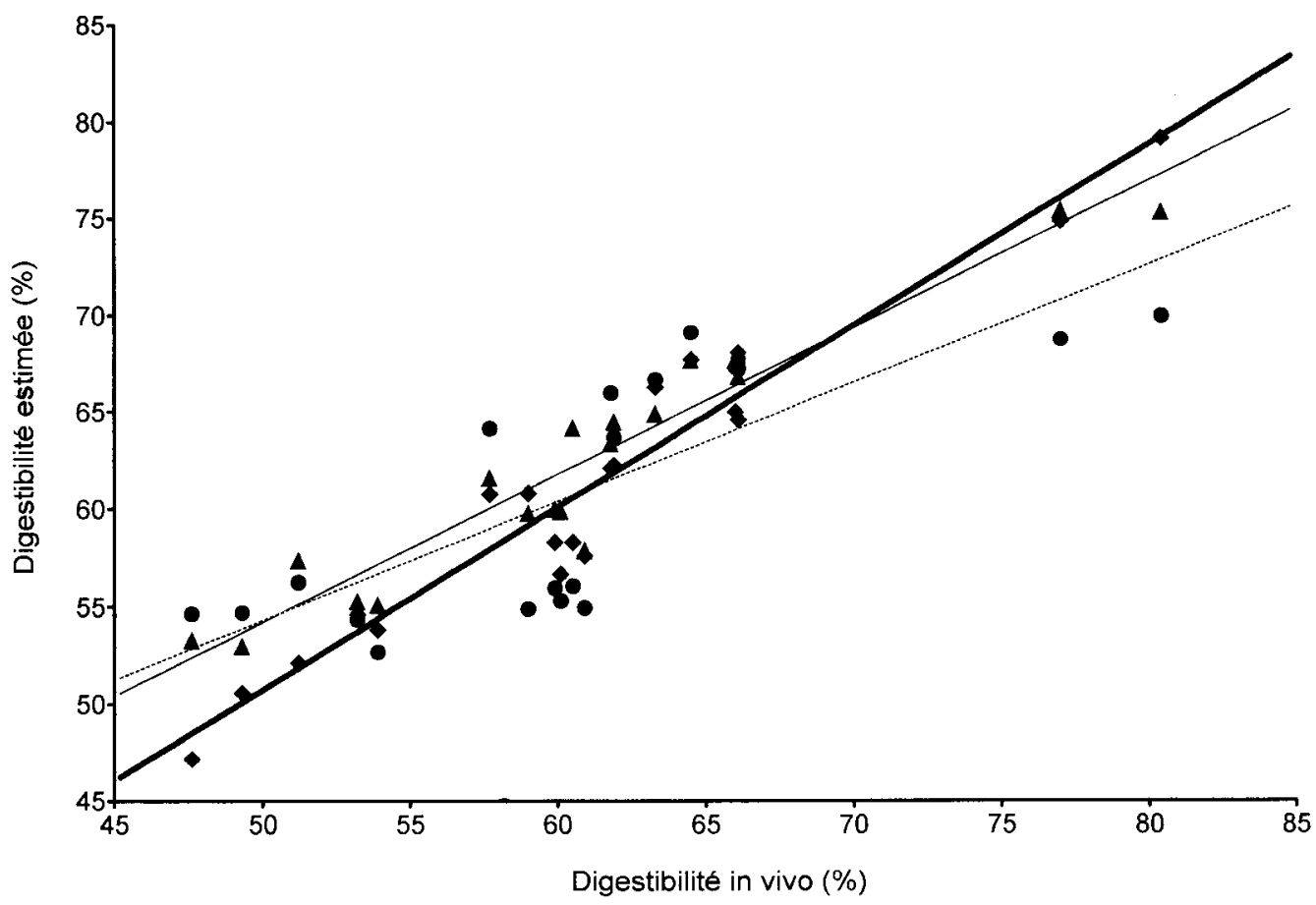

Fig 4. Utilisation de l'IANP comme critère de laboratoire pour l'estimation de la digestibilité de la matière organique des fourrages. $-\mathbf{A}-$ DMO enzymatique $\left(\mathrm{r}^{\wedge} 2=0,92\right) ; \cdots \cdot \mathrm{DMO}$ "Weende» $\left(\mathrm{r}^{\wedge} 2=0,61\right) ; \longrightarrow-\mathrm{DMOL}^{*}+\operatorname{IANP}\left(\mathrm{r}^{\wedge} 2=0,95\right)\left({ }^{*} \mathrm{DMOL}\right.$ voir tableau II $)$.

tion. Cette activité peut montrer, à concentrations comparables, des différences très importantes (Scehovic, 1990), dépendantes de l'espèce végétale, de son origine (sélectionnée ou naturelle), de la rapidité de croissance et de divers paramètres agronomiques. L'évaluation de l'«activité anti-biologique" des structures chimiques présentes dans des fourrages peut contribuer fortement à l'amélioration des performances des méthodes de laboratoire, mais aussi concourir à une meilleure compréhension de certains phénomènes dans le domaine des relations plante-animal.

Un certain nombre de tests dans un milieu microbiologique est en cours de réalisation dans notre laboratoire pour étayer les observations obtenues dans un milieu enzymatique.

\section{RÉFÉRENCES}

Aufrère J (1982) Étude de la prévision de la digestibilité des fourrages par une méthode enzymatique. $A n n$ Zootech 31, 111-130

Cheng KJ, Fay JP, Howarth RE, Costerton JW (1980) Sequence of events in the digestion of fresh legume leaves by rumen bacteria. Appl Environ Microbiol 40, 613-625

Barry TN (1989) Condensed tanins: their role in ruminant protein and carbohydrate digestion and possible effects upon the rumen ecosystem. In: The Roles of Protozoa and Fungi in Ruminant Digestion (JV Nolan, RA Leng, DI Demeyer, eds), Pehambul Books, Australia

Beart JE, Lilley TH, Haslam E (1985) Plant polyphenols-secondary metabolism and chemical defence: some observations. Phytochemistry 24 (1), 33-38

Borneman WS, Akin DE, Vaneseltine WP (1986) Effect of phenolic monomers on ruminal bacteria. Appl Environ Microbiol 52 (6), 133†-1339

Chesson A, Stewart CS, Wallace J (1982) Influence of plant phenolic acids on growth and cellulolytic acti- 
vity of rumen bacteria. Appl Environ Microbiol 44, 597-603

Daiber KH (1975) Enzyme inhibition by polyphenols of sorghum grain and malt. J Sci Fd Agric 26, 13991411

Griffiths DW, Jones DIH (1977) Cellulase inhibition by tannins in the testa of field beans. J Sci Fd Agric 28 , 983-989

Hagerman AE, Butler LG (1991) Tannins and lignins. In: Herbivores: their interaction with secondary plant metabolites (GA Rosenthal, MR Berenhoum, eds). Academic Press, Vol 1 (2nd edition) 355-388

Hartley RD, Jones EC, Fenion JS (1974) Prediction of the digestibility of forages by treatment of their cell walls with cellulolytic enzymes. J Sci Fd Agric 25, 947954

Hartley RD, Jones EC (1977) Phenolic components and degradability of cell walls of grass and legume species. Phytochemistry 16, 1531-1534

Jones DIH, Hayward MV (1973) A cellulase digestion technic for prediction the dry matter digestibility of grasses. J Sci Fol Agric 24, 1419-1426

Jung HJG (1988) Inhibition potential of phenolic-carbohydrate complexes released during ruminal fermentation. J Agric Food Chem 36, 782-788

Mueller-Harvey I (1989) Identification and importance of polyphenolic compounds in crop residues. $I n$ : Physico-chemical characterisation of plant residues for industrial and feed use (A Chesson, ER Orskov, eds), Elsevier Applied Science, NY, États-Unis, 88107

Scehovic J (1979) Prévision de la digestibilité de la matière organique et de la quantité ingérée des Graminées, sur la base de leur composition chimique. Fourrages 79, 57-78

Scehovic $\mathrm{J}$ (1988) Métabolites secondaires de quelques plantes de prairies. Recherche Agronom Suisse 27 (2), 153-165

Scehovic J (1990) Tanins et autres polymères phénoliques dans les plantes de prairies : détermination de leur teneur et de leur activité biologique. Revue Suisse Agric 22, 179-184

Scehovic J (1991) Considération sur la composition chimique dans l'évaluation de la qualité des fourrages des prairies naturelles. Revue Suisse Agric 23, 305310

Singleton VL, Kratzer FH (1969) Toxicity and related physiological activity of phenolic substances of plant origin. J Agric Food Chem 17, 497-512

Swain T (1977) Secondary compounds as protective agents. Ann Rev Plant Physiol 28, 479-501

Swain T (1979) Tannins and lignins. In : Herbivores: their interaction with secondary plant metabolites (GA Rosenthal, DH Janzen, eds), Academic Press, 657-682

Van Soest T (1981) Limiting factors in plant residues of low biodegradability. Agric Environ 6, 135-143 